\title{
ZEITSCHRIFT
}

FÜR

\section{KRYSTALLOGRAPHIE UND}

\section{MINERALOGIE}

UNTER MITWIRKUNG

ZAHLREICHER FACHGENOSSEN DES IN- UND AUSLANDES

HERAUSGEGEBEN

voN

P. GROTH.

\section{VIERUNDRDEISSIGSTER BAND.}

MIT 10 LITHOGRAPHIRTEN TAFELN DND 197 FIGUREN IM TEXT.

\section{LEIPZIG}

VERLAG VON WLHELM ENGELMANN

1901. 
